\title{
Heterogeneous Network Access for Seamless Data Transmission in Remote Healthcare
}

\author{
Piyush Yadav ${ }^{1 *}$, Rajeev Agrawal ${ }^{2}$ and Komal Kashish ${ }^{3}$ \\ ${ }^{1,2}$ Department of Electronics and Communication Engineering, G L Bajaj Institute \\ of Technology and Management, Greater Noida, India \\ ${ }^{3}$ RWTH Aachen University, Templergraben 55, 52062 Aachen, Germany \\ ${ }^{1}$ piyushyadav1985@gmail.com, ${ }^{2}$ rajkecd@gmail.com ${ }^{3}$ kashishkomal@ymail.com
}

\begin{abstract}
In Remote Health care application, the availability of reliable communication channel is a major challenge for successful transmission of the medical data from one point to other. Heterogeneous network functions across multiple radio technologies including Wireless Body Area Networks (WBANs). However, there is always a need of a model where the best suited network satisfying the need of transmitting the critical data is available to the user from a set of network. The paper presents an efficient and seamless Network selection scheme designed specifically for the transmission of a patient's medical data across multiple technologies. Further, the selected network through suggested scheme is tested on multiple Quality of Service $(Q o S)$ parameters such as Delay, Packet Error Rate (PER), Received Signal Strength (RSS), Signal to noise ratio (SNR), Data Rate and cost. The simulation studies establish the effectiveness of the suggested framework by selecting a network which is best suited as per the user's applications and type of medical data transmitted.
\end{abstract}

Keywords: Network Selection; Heterogeneous Network; Telemedicine; Wireless Body Area Network; Quality of Service

\section{Introduction}

As per the World Bank report the number of medical practitioners per 1000 people in the developed countries ranges between 2.1-2.7 where as in case of developing countries it is even worse. In India the health care system requirement is huge due to its massive population. By no means can we reach the minimum norms set by WHO i.e., 1:1000 from the present ratio of 1:1674 [1]. This is predominantly due to the high demand for doctors, which is further increasing at a very fast rate. However, there are many factors contributing to such a rise such as ageing population, increased life span of the people, polluted environment, urbanization and many other local and global factors. This problem becomes more severe in rural areas, as the major concentrations of physicians are in the metro cities, rural and remote areas are left with very few doctors [2].

The technologies in such cases can play a major role in providing an effective solution to these problems. Through information technologies one can think of extending the reach of healthcare services virtually to any remote part of the globe. To envisage the cases, one can have its medical consultations through voice or video calls, diagnose through transfer of medical data of a patient's over the network to the doctor is also possible. Such healthcare framework through a high quality video conference system extends the facility for patients to interact with doctors from a

Received (March 29, 2018), Review Result (May 14, 2018), Accepted (May 24, 2018) 
remote location / homes. In addition, various medical sensors can be installed at the patient's home in order to collect real time medical data [3].

However, telemedicine technology has come a long way since its initial establishment in $20^{\text {th }}$ century. It has witnessed a major growth with the exponential advancement in the area of communication and related technologies like multiple access networks and multimode terminals. As the communication technology has shifted from Global System for Mobile Communication (GSM) to a newer means such as Wireless Local Area Network (WLAN), Worldwide Interoperability for Microwave Access (WiMAX), Long Term Evolution (LTE) and Universal Mobile Telecommunications Systems (UMTS), it has become possible to transmit data at faster rate with least error, from one place to another [4]. With the advancement in Wireless technology any user without any physical connection can access ubiquitous data and telephony from anywhere at any time. The data could be Electrocardiograms (ECG), medical resonance images, and other vitals of a patient in case of healthcare monitoring [5]. However, similar to other applications using communication technologies, remote healthcare monitoring will also face the major challenges of connecting always, anywhere and anyone (3A) within the network. Further it is also a major challenge to select and implement the best suited network which can support the requirement of bandwidth, data rate and Received signal strength for such crucial application, none of the available remote access techniques can alone fulfill the wide diversity of channel and network requirements [6].

This attracts the designing and implementation of a network selection scheme in a heterogeneous network environment which can select the best suited or optimum network as per the user choice or need for an error free and reliable transmission of medical data. With this paper, we aim to make the following contributions;

- A non-complex and yet efficient system for seamless switching

- The system should be able to decide on the suitability of the networks for any particular class of data as per its QoS requirements

- Minimization of handovers

- To suggest a network selection algorithm for the transmission of medical data, taking into consideration the importance of the class of service data.

- The selection of the network is done so as to provide an efficient patient monitoring system in a heterogeneous environment

The paper is divided into different sections with Section 2 gives the details of the related works. The system framework used for Network selection in wireless heterogeneous environment is explained in Section 3. Section 4 elaborates the proposed network selection scheme and its algorithm. The performance metrics used for network selection is also obtained for better understanding of the framework. Performance evaluation of proposed algorithm is carried in Section 5. Last section draws the conclusion of the paper.

\section{Related Work}

In this section we are mentioning about the different recent works and surveys dealing with heterogeneous network selection and handover phenomenon that are related to our work.

Various radio technologies such as WLAN, WiMAX and cellular networks have been employed for data transmission in telemedicine. In this context, the design suggested in $[7,8]$ uses WLAN for monitoring patients in a hospital environment. In [9], the similar design is suggested by using WiMAX in healthcare. However, using 
only one technology for such a layout has some of its limitation such as the issue of limited cell coverage in WLAN or lower bandwidth in cellular technologies not suitable for certain telemedicine services requiring high resolution transmission.

In [10] it was then concluded that the varying problems of homogenous networks could be overcome by a network selection method in a heterogeneous network environment which helps in selecting the most suitable network depending on the requirements of the data to be transmitted. Authors in [11, 12] has extensively elaborated the reliability, fault tolerance and Transmission delay paradigms suggested for WBANs. In [13] a mobile WBAN-based environment with all the Technological aspects of WBANs for health monitoring is discussed.

The work proposed in [14] explains about RSS based vertical handover decision and gives the comparison among the obtained RSS values by mobile terminals. However, selection mechanism fall low on complexity scale by basing the decision on only one parameter and are less effective when compared with the methods where the decision depends on single or multiple attributes such as Cost, RSS, SNR, User Preferences and many more as proposed in [15]. In [16] authors have combined various parameters such as the Bandwidth, link quality, Energy consumption and security aspects in normalized weighted function to select the best network available. In [17] the handover decision between WiMAX and WiFi is done based on cost function. In regions which have both kinds of network available the network with low cost is preferred.

In [18,19] Multi Attributes Decision Making (MADM) techniques are implemented through quantitative calculations done using multiple attributes of the available networks, presents performance evaluation between multi-attribute methods such as Simple Additive Weighting (SAW), Multiplicative Exponent Weighting (MEW), Grey Relational Analysis (GRA), Technique for Order Preference by Similarity to Ideal Solution (TOPSIS), Preference Ranking Organization Methods for Enrichment Evaluations (PROMETHEE), Elimination Et Choice Translating Reality (ELECTRE).

Authors in [19] also reported the comparison between different wireless technologies (i.e., IEEE $802.11 x$, UMTS, LTE) and proposes a new but effective multi-attribute decision making handover approach for WBAN. Further several mechanisms of handover have been proposed to design and solve the problem. However, it was observed that for remote healthcare services no effective solution has been proposed taking into the consideration the QoS aspects needed for such application.

\section{Proposed Framework for Network Selection}

As discussed in previous section the issue is to select the appropriate network which can able to provide the required QoS for successful transmission of patient's medical data. The selected wireless network has also provides a seamless connectivity with minimum delay to guarantee the delivery of the packet (Information) at the receiving end.

The six modules of the proposed framework are, i) WBAN Information Collection and network Discovery (ii) Network QoS Parameters Evaluation as per medical data (iii) Medical Data Categorization (iv) Network Monitoring Based on QoS Parameters (v) Determination of Network Score and Decision Parameters, (vi) Network Selection.

The proposed framework follows the steps as shown in Figure 1, to select an appropriate network as per the need. 


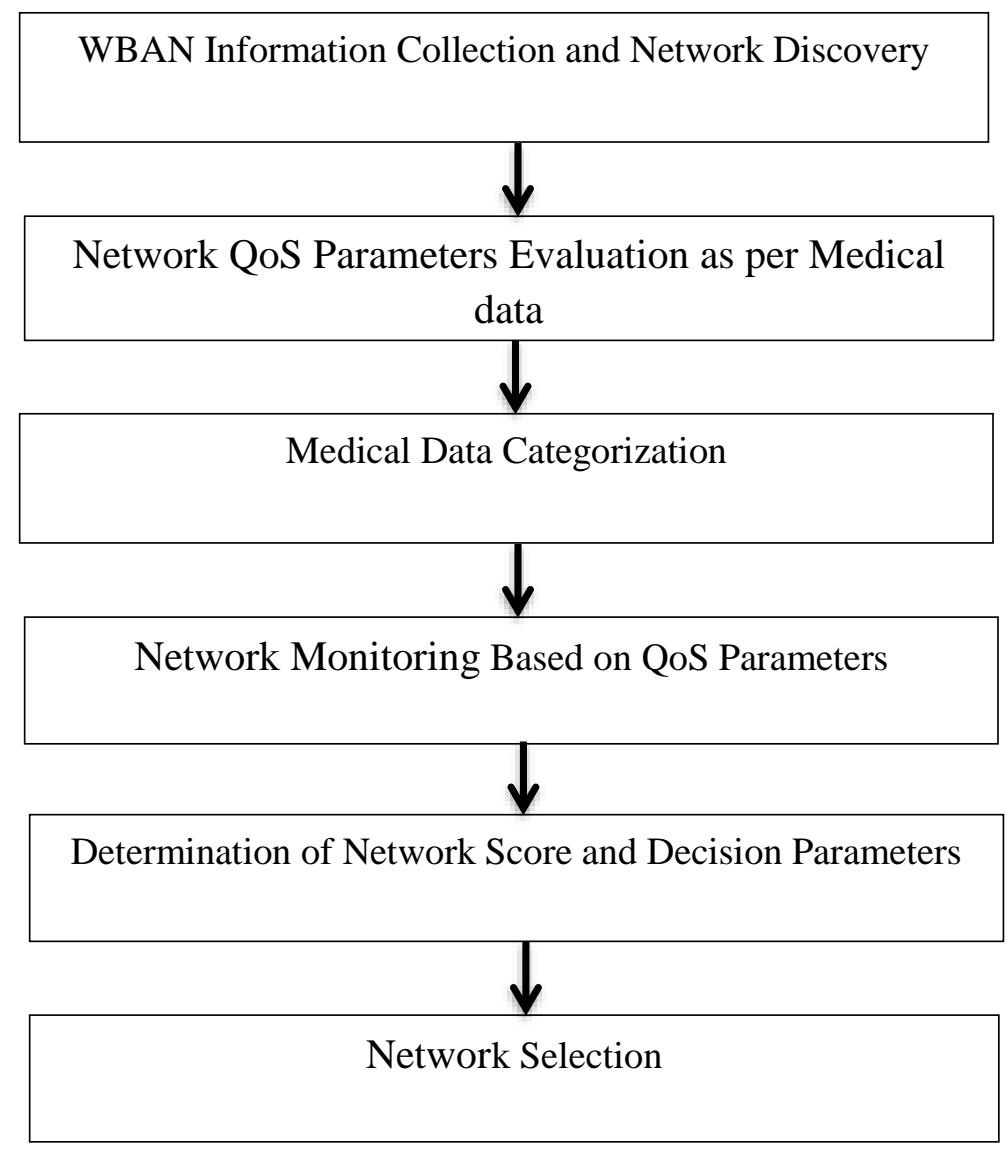

Figure 1. Proposed Framework for Network Selection

\subsection{WBAN Information Collection and Network Discovery}

We have considered a patient with a well-functioning WBAN device equipped to collect, store and forward acquired data to next set of network [19]. The device is responsible to maintain the reliable communication between the sensors on the body and interfaces along with forwarding the vital body parameters collected, to be transferred to the next level of network. However, this next level of network is a heterogeneous network comprising of different networks such as WLAN, WiMAX, etc. Anyhow, the collected data from all the sensors is to be transmitted to destined node so that the necessary medication is to be extended to the patient. A handover process is to be initiated between the device (on the body) and heterogeneous network to establish a reliable connection.

Whenever a handover is initiated in a particular area Mobile Terminal (MT) discovers the available networks within its coverage area. It collects the information for the discovered networks based on network conditions, user requirements. In our study, however, we have kept our focus on WLAN, WiMAX, LTE and UMTS. The reason behind selection of these four technologies for our framework was the growing trend of use of these wireless technologies in patient monitoring and other personal devices.

\subsection{Quality of Service Parameters}

To select the best suited network as per the need of the user, a system is required to have certain quality matrices on which all the available networks are examined 
and compared. For our proposed study we have taken the following Quality of Service (QoS) parameters which are widely used.

3.2.1. Received Signal Strength Intensity (RSSI): The RSSI here relates to the known transmission power of the communication technology. In simpler terms, low signal power gives low RSSI. In case of LTE, it is the measure of average power found in OFDM symbols containing reference symbols. RSSI is related to the distance between the nodes. i.e., longer distances correspond to lower RSSI. The RSSI value of any available network at any instance can be calculated as

$$
\text { RSSI } \left.=\mathrm{P}_{\mathrm{t}}-10 \eta \log \left(\mathrm{d} / \mathrm{d}_{\mathrm{o}}\right)-\text { fading effect (random variable }\right)
$$

Where $P_{t}$ is the power transmitted by the sender, $\eta$ is the path loss coefficient, $d$ is the distance between transmitter and receiver and $d_{O}$ is the reference distance used in far field conditions.

3.2.2. Delay: Delay might be a result multiple factors in communication technology, such as multipath propagation, channel capacity, geographical conditions etc. The latency of the technology used also varies the delay in the system. UMTS and $3^{\text {rd }}$ generation systems for examples have comparatively higher latency as compared to LTE systems.

LTE also triumphs over the other two technologies Wi-Fi and WiMAX discussed in the paper. Improvement in latency with time in these systems facilitate TCP uploads and downloads. Services discussed in this paper, especially VoIP or video conferencing are directly benefitted with decrease in latency of the technology used.

3.2.3. Data Rate: Data Rate is used to denote transmission speed of a network, or the number of bits transferred per second. Overall, the ideal data rate of any network for any service is higher than the bits actually transmitted. This is due to the need of additional bits for purposes such as addressing, error correction, signalling etc.

3.2.4. Packet Error Ratio (PER): PER is one of the important aspects which gives an estimate defined as the ratio of not successfully received packet to the total packet send to any destined node test set. It is used to test the performance of any access terminal's receiver. Further, packet is a unit for data for radio transmission which is subject to forward error correction (FEC).

3.2.5. Signal to Noise Ratio: SNR is the ratio of the maximum signal strength that a wireless connection can achieve to the noise present in the connection. The SNR of a network needs to be as high as possible. Higher the value of SNR, the better will be the signal strength and the quality of transmission.

The parameter or SNR is more related to the service requirement threshold than the technology. Channels which offer high SNR can be used for highly error sensitive service such as ECG or Electroencephalography (EEG), whereas services such as video conferencing can still function without significant issues with comparatively lower SNR than as required by ECG, EEG etc., at the benefit of having lower network cost.

3.2.6. Network Cost: Network cost is the total operational cost of the network. A fair trade-off between the network cost and service quality needs to be implemented to ensure that there is no loss of crucial patient data. For our analysis, we will take network cost into consideration according to the severity of the data. For example, in case of emergency services, the issue network cost is not considered to have 
much weight but in case of general monitoring data, network cost is to be considered while taking the final decision.

\subsection{Medical data Categorization}

For the implementation of the proposed framework, which can suggest the most suited network within the set of network, we have categorized the type of data (test) into four classes (1-4) where the basis of classification is the QoS requirement of that data type as presented in Table 1. This categorization is done based on the priority assigned to the clinical tests performed on the patient depending upon the severity of the cases. For example, if a patient is unconscious and is under a critical care, the type of diagnosis and test performed will be different from a case where the life of a patient is not under threat. In our proposed framework, the priority of such test will be higher compared to the normal tests and comes under Class I. Further, the QoS parameters to be analysed during transmission of such data (ECG/EEG) are data rate, error rate, network cost, received signal strength intensity and delay. Each parameter associated with the type of particular class is assigned a score which gives a semantic ranking in terms of very high, high, medium, low and very low, which is optimum requirement for transmission of medical data successfully from one location to another.

The value $\mathrm{v}^{\mathrm{m}}$ reflects the level of priority of the given parameter for the service class in use. This score of networks, $\mathrm{v}^{\mathrm{m}}$ is assigned as per the requirement of data parameter $[19,20]$ in our framework. Here, Class 1 holds the highest priority while Class 4 belonging to the services such as sensory data has the lowest. As discussed in the earlier section, this priority is decided upon the severity of the clinical state in which particular services are required.

Table 1. Level of Priority $\left(\mathrm{v}^{\mathrm{m}}\right)$

\begin{tabular}{|c|c|c|c|c|}
\hline $\begin{array}{c}\text { Parameters (m) } \\
\text { Service } \downarrow\end{array}$ & RSSI & Delay & Data rate & $\begin{array}{c}\text { Packet Error } \\
\text { Ratio }\end{array}$ \\
\cline { 1 - 3 } $\begin{array}{c}\text { Class 1 (ECG, } \\
\text { EEG) }\end{array}$ & High (0.8) & Medium & High & Very High \\
(1)
\end{tabular}

Network Parameter Requirements of different service classes is shown in Table 2 [18], the priority in Table 1 is assigned by the value of parameters in Table 2. Using Table 2, the weight, $\mathrm{w}^{\mathrm{m}}$ of each parameter for all the available networks is calculated using entropy method [22] which contributes in the calculation of another parameter, score ${ }^{\mathrm{m}}$ (see section 3.5). Once the $\mathrm{v}^{\mathrm{m}}$, score ${ }^{\mathrm{m}}$ and priority (class) is set for the parameter, the selection of best suited network is obtained. 
Table 2. Network Parameter Requirements of Different Service Classes

\begin{tabular}{|c|c|c|c|c|c|c|c|c|c|}
\hline \multirow{2}{*}{$\begin{array}{c}\text { Class } \\
\quad \downarrow\end{array}$} & \multirow{2}{*}{$\begin{array}{c}\text { Parameter } \\
\rightarrow\end{array}$} & \multicolumn{2}{|c|}{ Delay (s) } & \multicolumn{2}{|c|}{ RSSI (dB) } & \multicolumn{2}{|c|}{ Data Rate (kbps) } & \multicolumn{2}{|c|}{ PER } \\
\hline & & Min & $\operatorname{Max}$ & Min & Max & Min & Max & Min & $\operatorname{Max}$ \\
\hline Class 1 & & 6 & 9 & -50 & -40 & 300 & 400 & 0 & $10^{-6}$ \\
\hline Class 2 & & 7 & 10 & -55 & -40 & 280 & 400 & 0 & $10^{-6}$ \\
\hline Class 3 & & 4 & 8 & -65 & -50 & 400 & 700 & $10^{-5}$ & $10^{-4}$ \\
\hline Class 4 & & 85 & 100 & -85 & -65 & 40 & 110 & $10^{-4}$ & $10^{-2}$ \\
\hline
\end{tabular}

Now, to utilize the classification of data presented in this section, the next section follows up with a network monitoring based on QoS parameters required for each class.

\subsection{Network Monitoring based on QoS parameters}

Network monitoring is done to check the minimum QoS parameters which are required for a particular application. To ensure that no critical data is lost due to network connectivity, a safety factor is taken where the network conditions of the selected network are above the desired threshold. The values of network parameters are taken as per the Media Independent Handover (MIH) standard (IEEE 802.21) [21]. MIH standard enables seamless switching between two different wireless technologies and maintain interoperability between them.

The network suitability is monitored usually taking into consideration RSS value of the network currently in use. The decision to switch network is made on the basis of the RSS value compared to the threshold RSS value. However, RSS alone cannot be the only factor on which the decision of switching should be based as other QoS parameters such as the delay, data rate of the network, bandwidth etc., are also an important factor when determining whether a network is still apropos to remain functioning for providing the service to the specified class of service.

Hence, for the monitoring purpose in the proposed model, we have included the network parameter, data rate of the network which is defined as,

Data rate $=B W^{*} \log _{2}(1+S N R)$,

Where BW is the bandwidth offered by the network and SNR denotes the signal to noise ratio in any network.

If the value of either RSSI or Data rate falls below the decided threshold, then a switching process is triggered and the framework steps into the fifth phase that is to decide the score of all the available networks.

\subsection{Determination of Network Score and Decision Parameter}

Once the request for switching is triggered, the system collects the value of all the QoS parameters on which the decision is to be based. With all the values acquired, a network matrix is created. 
$\mathrm{u}^{\mathrm{i}}=\left|\begin{array}{ccccc}u_{11}^{i} & u_{12}^{i} & u_{11}^{i} & \ldots & u_{1 m}^{i} \\ u_{21}^{i} & u_{22}^{i} & u_{23}^{i} & \ldots & u_{2 m}^{i} \\ u_{31}^{i} & u_{32}^{i} & u_{33}^{i} & \ldots & u_{3 m}^{i} \\ \ldots & \ldots & \ldots & \ldots & \ldots \\ u_{n 1}^{i} & u_{n 2}^{i} & u_{n 3}^{i} & \ldots & u_{n m}^{i}\end{array}\right|$

where $\mathrm{i}$ is the class of service selected (Class 1, Class 2 etc.), $\mathrm{n}$ is the total number of networks available to engage in switching process and $\mathrm{m}$ is the total number of network parameters on which the decision of switching would be based.

Now for any parameter $\mathrm{m}$, Let $u_{n m}^{i}=\mathrm{a}^{\mathrm{m}}$

Where $\mathrm{a}^{\mathrm{m}}$ is the present value of that network parameter.

Every service class, network parameters have a min and max threshold value [Table 2], such that $a_{\min }^{m}$ is the minimum threshold value of that parameter for a particular class and $a_{\max }^{m}$ is the maximum threshold value of that parameter.

Now, the parameter value is normalized using the max-min normalization technique

$f^{m}=\left\{\begin{array}{cl}0 & \text { for } a^{m}<a_{\min }^{m} \\ \frac{a_{\text {value }}^{m}-a_{\min }^{m}}{a_{\max }^{m}-a_{\min }^{m}} & \text { for } a_{\min }^{m}<a^{m}<a_{\max }^{m} \\ 1 & \text { for } a^{m}>a_{\max }^{m}\end{array}\right.$

Here $\mathrm{f}^{\mathrm{m}}$ is the normalized value of the acquired network parameter which fall in the range of min and max threshold value. Further, for a particular parameter $\mathrm{m}$ the weight of the parameter is determined using the entropy weighting method. Entropy and the normalized value of the parameter are related by the following expression [22].

$E^{m}=\frac{-\sum_{i=0}^{n} f_{i}^{m} * \ln f_{i}^{m}}{\ln (n)}$

Where $\mathrm{E}^{\mathrm{m}}$ is the entropy value of the parameter, $\mathrm{n}$ is the total number of networks available and $\mathrm{f}^{\mathrm{m}}$ is the normalized parameter value from Equation 1.

An entropy based weight factor is determined for every parameter value such that,

$w^{m}=\frac{1-E^{m}}{\sum_{i}^{m}\left(1-E^{m}\right)}$

where $\mathrm{w}^{\mathrm{m}}$ is the weight of the network parameter.

Now, using this $\mathrm{w}^{\mathrm{m}}$, we finalize a parameter score ${ }^{\mathrm{m}}$ of the parameter such that

$\operatorname{score}^{m}=f^{n *} w^{m}$

Using Table 1, priority $\mathrm{v}^{\mathrm{m}}$ for the networks are calculated.

$v^{m}=\left[\begin{array}{llll}0.8 & 0.6 & 0.8 & 1.0 \\ 0.8 & 0.6 & 0.8 & 0.8 \\ 0.6 & 0.8 & 1.0 & 0.8 \\ 0.4 & 0.2 & 0.4 & 0.6\end{array}\right]$

Finally, for any particular network a decision parameter D $(n, i)$ is calculated by the summation of all the product of $\mathrm{v}^{\mathrm{m}}$ and score ${ }^{\mathrm{m}}$ values of all its $\mathrm{m}$ parameters.

$D(n, i)=\sum_{x=1}^{m} v^{m} * \operatorname{score}^{m} \quad$ [For Network $\mathrm{n}$ and Class i] 
A decision matrix is designed according to the decision parameter of all the networks and a network priority list is created.

$\mathrm{D}^{\mathrm{i}}=\left|\begin{array}{c}D_{1}^{i} \\ D_{2}^{i} \\ D_{3}^{i} \\ \cdots \\ D_{n}^{i}\end{array}\right|$

\section{Proposed Algorithm for Network Selection}

The objective of our algorithm is to determine the most suitable network for the class of medical data to be transmitted among a list of available networks. The proposed algorithm consists of the following steps

Step 1: Determine Class of Service

Step 2: Regular monitoring of RSS value of the current selected network. If the required value drops below the threshold limit, switching process is triggered

Step 3: A switching request can also be triggered by the users manually if they want to switch over to some other network

Step 4: QoS parameters of all the available networks are collected and separated into parameter list ( $\mathrm{m}$ is the no. of parameters list, i.e., there are total $\mathrm{m} \mathrm{QoS}$ parameters which are used in decision making. Here, we have $\mathrm{m}=5$ )

Step 5: According to the need of segregated data classes, every list is assigned a point $v^{m}$ from 0 to 1

Step 6: Now, our proposed alteration is to use another factor called $s c o r e^{m}$ to each member of every list. Here $\mathrm{i}$ denotes the class level (Class i). score $^{m}$ is calculated from score $e^{m}=f^{n} * w^{m}$ where $\mathrm{w}^{\mathrm{m}}$ is the entropy based weights of the factors calculated with equations 2 and 3 .

Step 7: For every network n, a Decision Parameter $[D(n, i)]$ is calculated from the following

$$
D(n, i)=\sum_{x=1}^{m} v^{m} * \operatorname{score}^{m} \quad \text { [For Network n and Class i] }
$$

Step 8: A rank list is prepared according to the value of Decision Parameter. Larger the value of Decision Parameter, higher the network is on the priority list.

Step 9: Channel availability for the network priority network is checked. If available, the Authentication Unit in the system facilitates a key exchange for the session. This is a common model for all the networks. It is required to ensure network security

Step 10: If the key authentication is successful, the switching occurs. Else, it moves to the next network option in the priority list.

To summarize, our proposed network selection approach is outlined in the flowchart depicted in Figure 2 


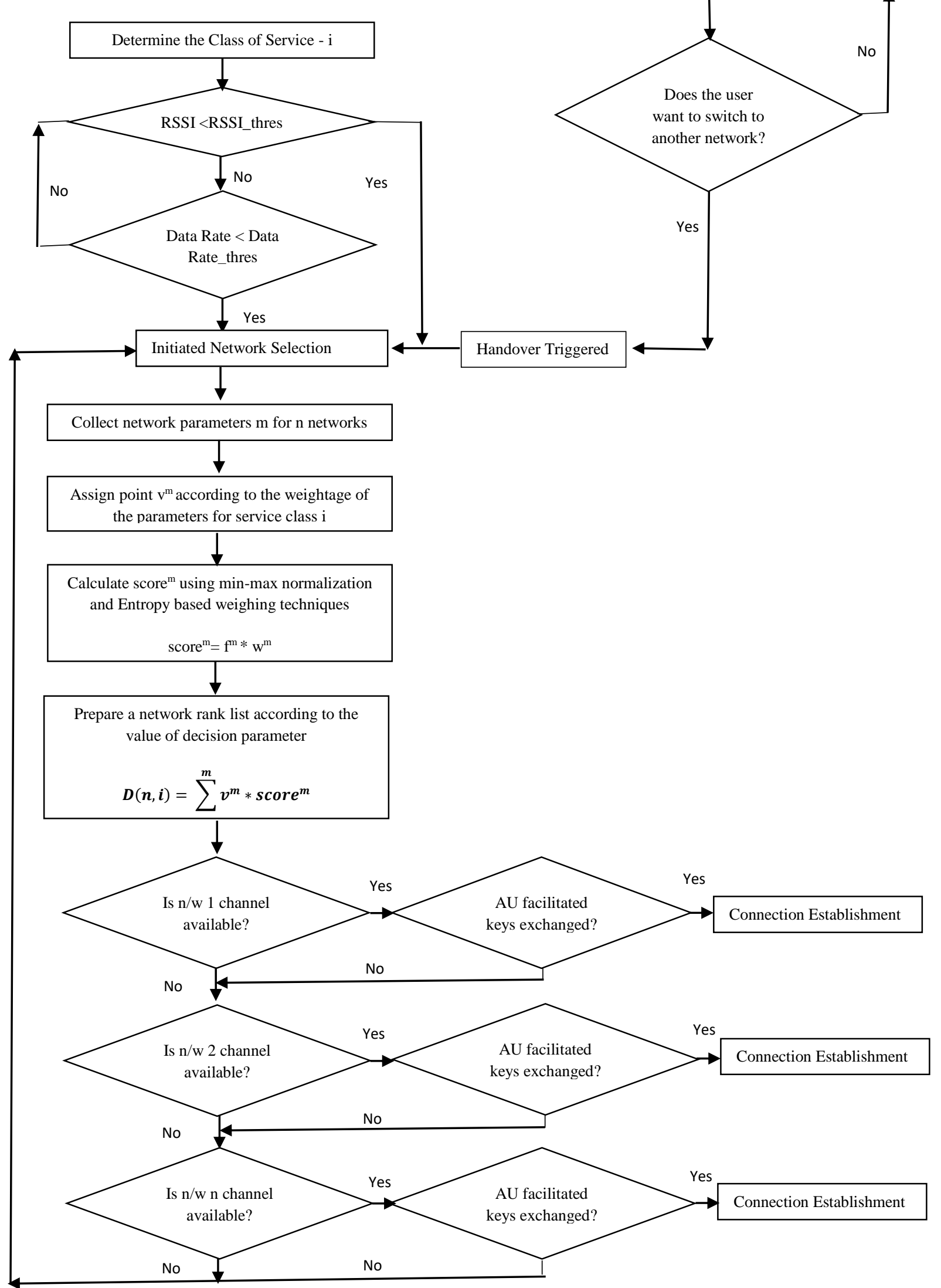

Figure 2. Flow Graph of Proposed Network Selection Scheme 


\section{Performance Evaluation}

To evaluate the performance of our proposed work simulation were carried out and the numerical values are obtained. Simulation was focussed to test the algorithm for different classes of data.

In the following sub-sections, we have described the network setup used in our simulation and then discuss the performance behaviour as per the proposed algorithm.

\subsection{Network Setup}

To test our proposed algorithm, it was required to setup an environment with network parameters assigned to the test networks. Table 3 lists out the parameters of the different networks to test the performance of our suggested algorithm.

Table 3. Network Simulation Parameters $[23,24]$

\begin{tabular}{|l|c|c|c|c|c|c|}
\hline $\begin{array}{l}\text { Wireless } \\
\text { Technology }\end{array}$ & $\begin{array}{c}\text { Received } \\
\text { Signal } \\
(\mathrm{dBm})\end{array}$ & $\begin{array}{c}\text { Delay } \\
(\mathrm{sec})\end{array}$ & $\begin{array}{c}\text { Data } \\
\text { rate } \\
(\mathrm{Mb})\end{array}$ & $\begin{array}{c}\text { Packet Error } \\
\text { Rate }\end{array}$ & $\begin{array}{c}\text { Bandwidth } \\
(\mathrm{MHz})\end{array}$ & $\begin{array}{c}\text { No. of user } \\
\text { requests processed } \\
\text { in one time unit }\end{array}$ \\
\hline WLAN & -50 & 4.8 & 10 & $26^{*} 10^{-4}$ & $20 \mathrm{MHz}$ & 10 \\
\hline WiMAX & -60 & 1.2 & 25 & $4.3^{*} 10^{-4}$ & $6 \mathrm{GHz}$ & 650 \\
\hline LTE & -55 & 0.2 & 15 & $10^{-6}$ & $10 \mathrm{MHz}$ & 200 \\
\hline UMTS & -70 & 2.5 & 2 & $10^{-5}$ & $5 \mathrm{MHz}$ & 129 \\
\hline
\end{tabular}

For our simulation setup, we took the scenario of a hospital with medical data from 100 patients being handled by a central nursing station established in a department of the hospital as shown in Figure 3. This central station is a way for both the nurses and doctors to keep an eye on the vitals of all the patients at once.

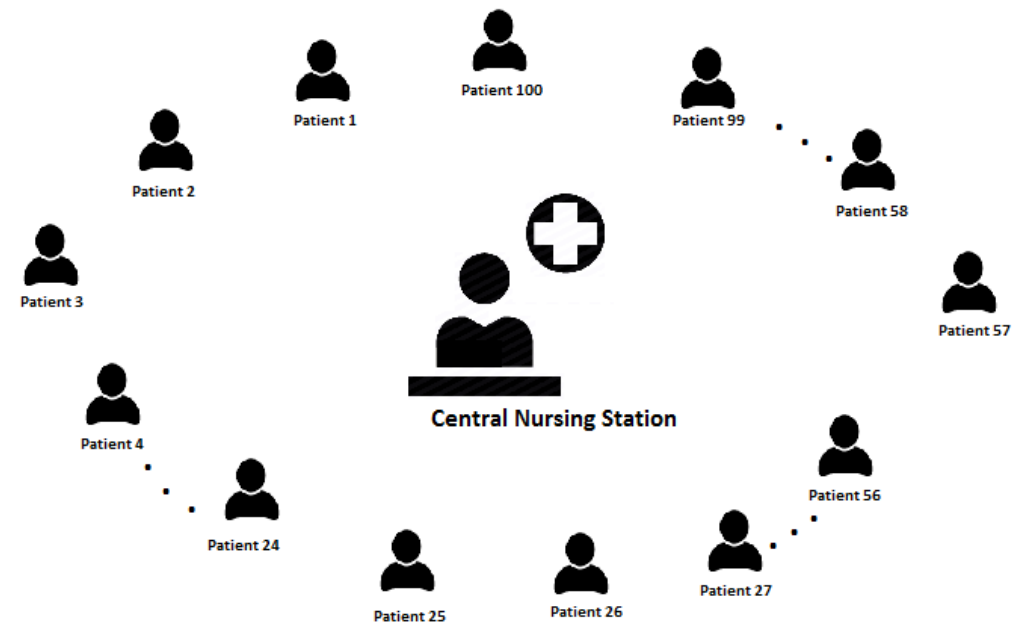

Figure 3. Simulation Setup

\subsection{Simulation Results and Discussions}

Simulations were carried to judge the efficacy of the proposed model. MATLAB was chosen as the tool of simulation because of its ease of use and wide availability of its resources. The network designed was of a hospital scenario with the 100 
patients or WBAN devices. In our simulation, we equipped our system to perform different calculations on the network data depending upon the class of data that is taken as input by the user.

\subsubsection{SNR Analysis}

Figures 4 to 8 show the SNR analysis done on all the technologies (LTE, WiMAX, WLAN and UMTS) that were used in our system. The results relate the SNR of the technologies with the data rate and bandwidth offered by them. A high SNR value is required for crucial data where no loss of data can be tolerated, hence an SNR analysis helps in concluding whether any technology is fit to be used in a critical environment such as that of telemedicine.

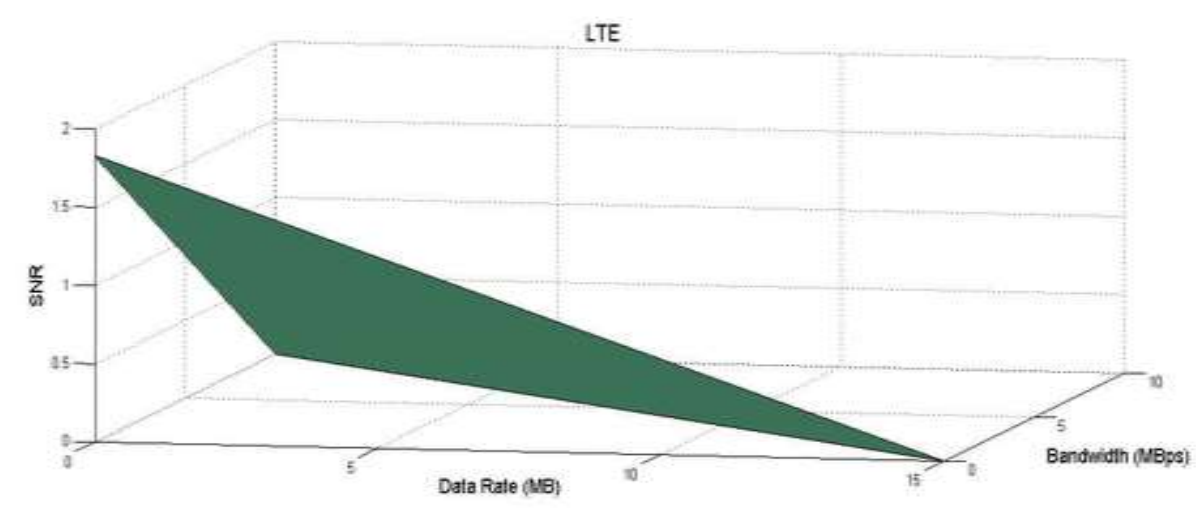

Figure 4. SNR Analysis of LTE

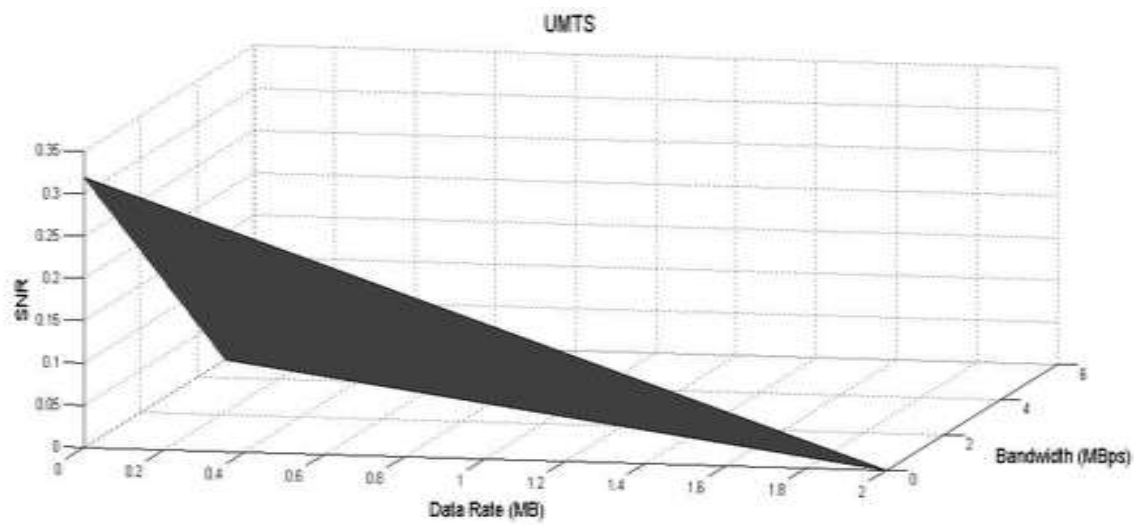

Figure 5. SNR Analysis of UMTS

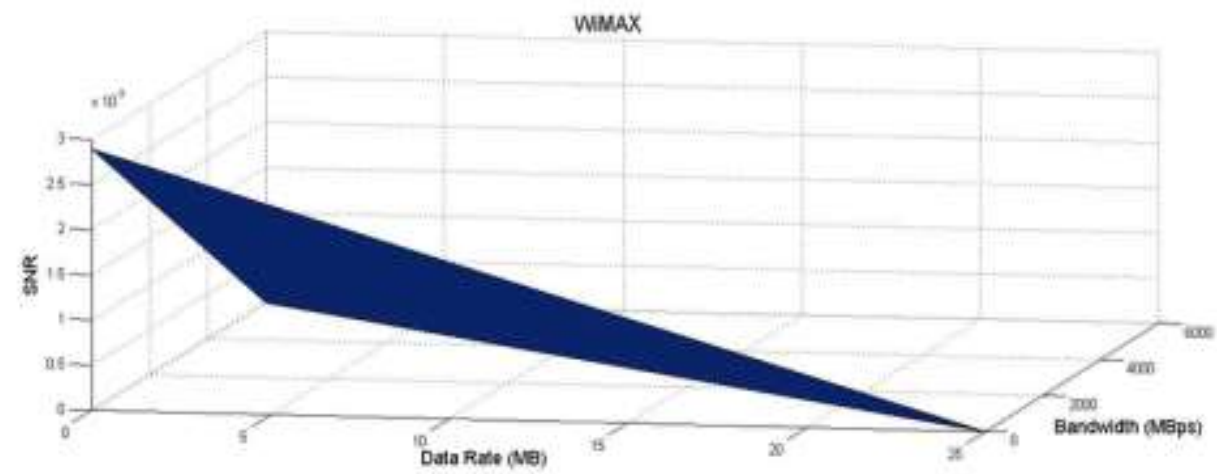

Figure 6. SNR Analysis of Wi-MAX 


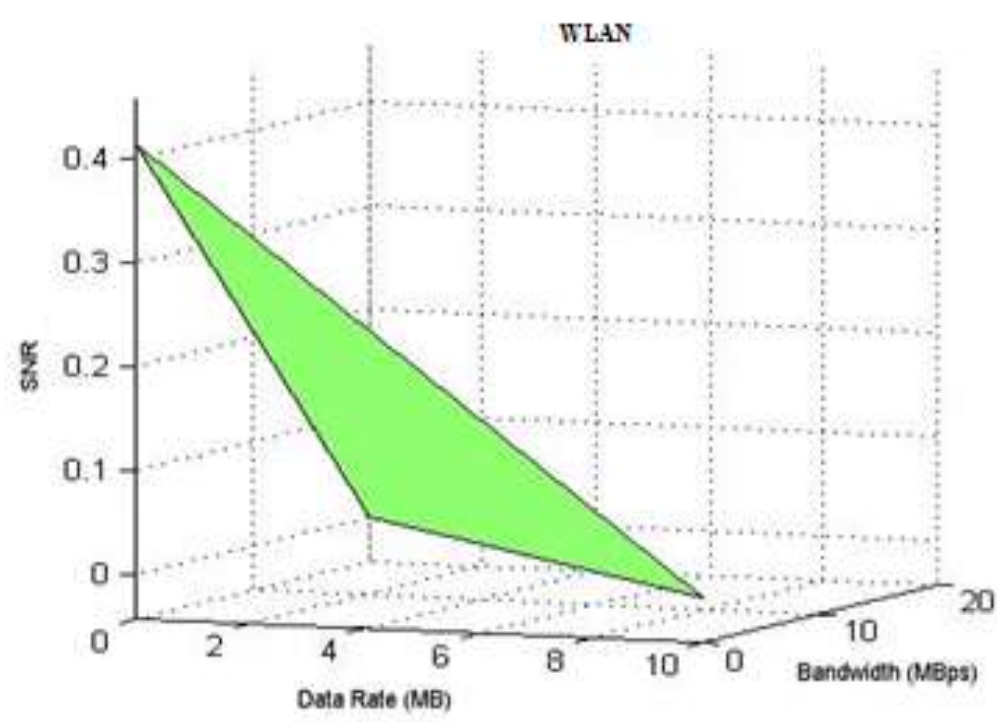

Figure 7. SNR Analysis of WLAN

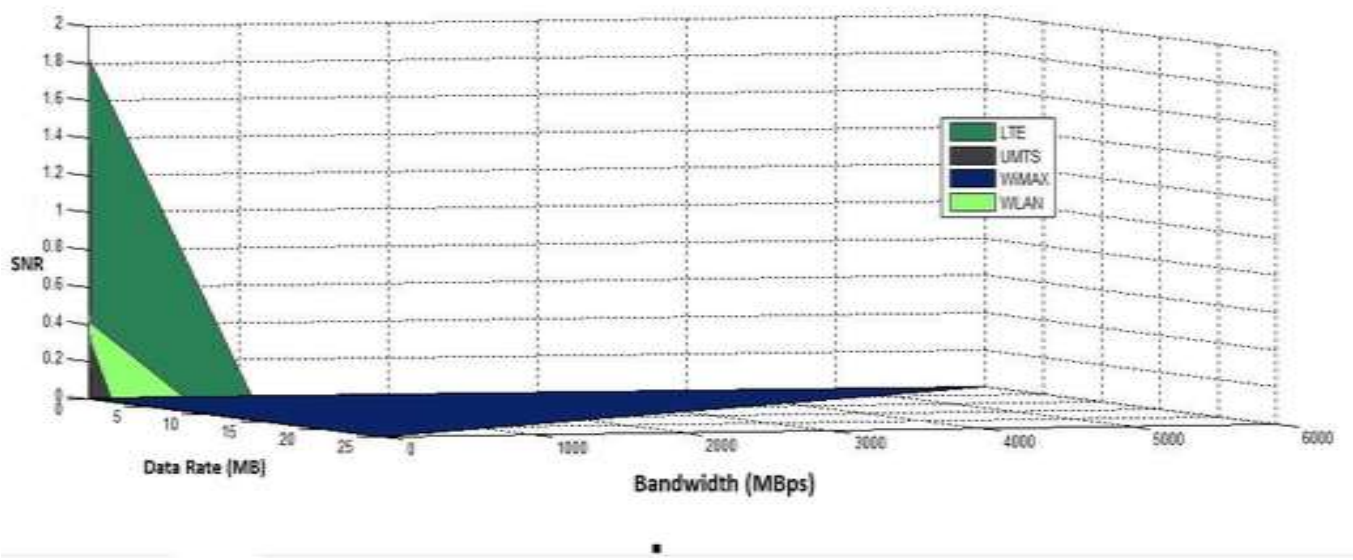

Figure 8. Combined Signal-to-Noise Ratio Analysis of LTE, UMTS, WiMAX and WLAN

From analysis we have observed that on average SNR of LTE is higher than the other three technologies while SNR of Wi-MAX is the lowest among all the options. High value of SNR means better signal strength and higher quality. SNR limits the number of symbols to be transmitted to make any system robust against unwanted noise, which is a highly crucial requirement in telemedicine data as loss or tamper of health vitals can result in adverse consequences. However, limiting the symbol rate affects bandwidth usage of the system. Hence, there has to be a trade-off between allocated bandwidth and signal to noise ratio. The importance of data rate factor depends upon the requirement of the telemedicine service. In a case, where the service has a high noise tolerance threshold but requires high bandwidth and data rate usage such as video conferencing or high definition videos, WiMAX is a viable option. In crucial systems, where the noise tolerance of the service is required to be minimal, LTE comes out as the best option. Table 4 presents a tabular representation of the comparative analysis of the four wireless technologies obtained from simulation results. 
Table.4 Comparative Analysis of Wireless Technologies

\begin{tabular}{|c|c|c|c|}
\hline $\begin{array}{c}\text { Wireless } \\
\text { Technology }\end{array}$ & SNR & $\begin{array}{c}\text { Bandwidth } \\
(\mathrm{Mb})\end{array}$ & Data Rate (Mbps) \\
\hline LTE & 1.8 & 10 & 15 \\
\hline UMTS & 0.32 & 5 & 25 \\
\hline Wi-MAX & .0028 & 6000 & 7.6 \\
\hline WLAN & 0.42 & 18 & 2 \\
\hline
\end{tabular}

\subsubsection{Number of Handovers}

The number of handovers executed by our framework was compared against a scenario of purely RSS based handover as shown in Figure 9. For this analysis, we considered WBAN devices of 100 patients which transfer data to a nursing station on regular intervals. The results derived here were assumed for a span of one hour.

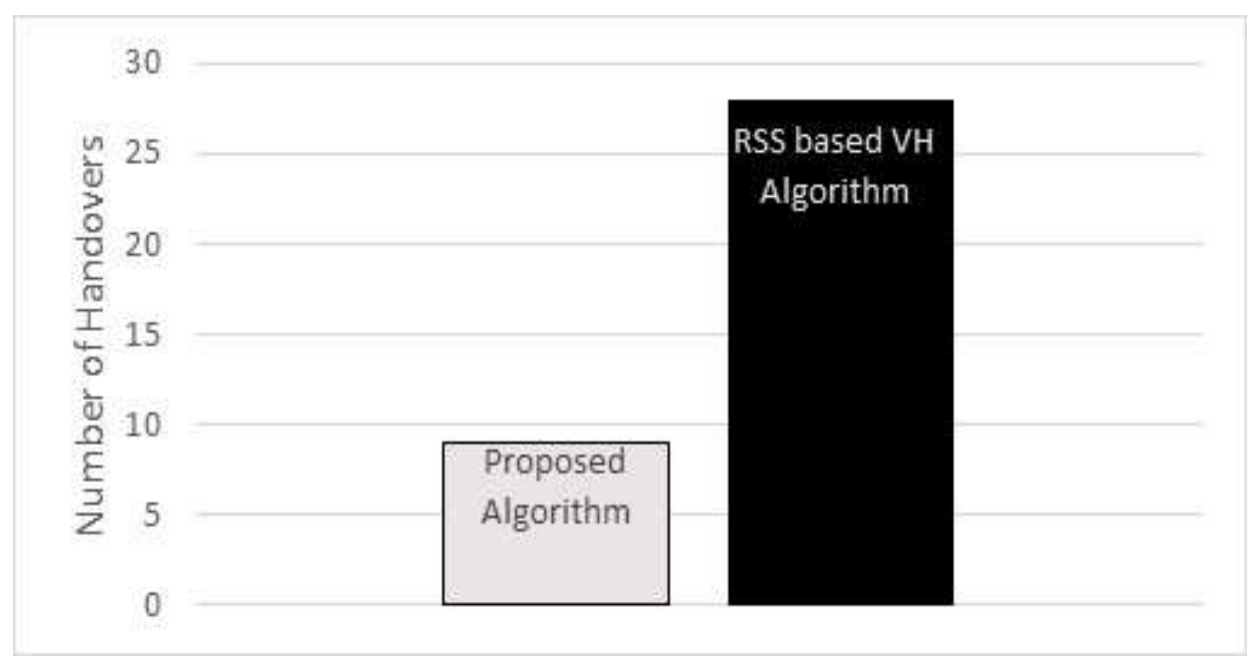

Figure 9. Number of Handovers

Within the simulated time, the WBAN devices first have to send the patients' sensory data such as blood pressure, heart rate value periodically. On larger intervals, ECG and EEG for some of the selected patients were also recorded and transmitted. However, for some other patients, services such as X-Rays and video transmission to a remote location were needed. In all the cases, the class of data changed multiple times and hence new more suitable networks were selected by the algorithm for each requirement. Compared to RSS based network switching, our algorithm as observed from Figure 9 was able to minimize the number of unnecessary switching, saving time and energy.

\subsubsection{Evaluation of Decision Parameters for Different Service Class}

Figure 10, shows the variation of Decision Parameter, $D(n, i)$ for all the classes when WLAN, WiMAX, LTE and UMTS networks are considered. As observed from the plots of service classes, the algorithm is able to decide which one of the available networks is the most suitable for the data to be transmitted. To evaluate the decision parameter, we consider the case of one of the patients out of the 100 assumed ones. The heart rate of the patient is sent over to the central nursing station every ten minutes along with the recorded blood pressure. For both blood pressure 
and heart rate data, the class of data is decided to be fourth type i.e., Class IV and for that particular class of data the algorithm suggests the use of LTE or WiMAX with these two networks having the highest value of Decision Parameter.

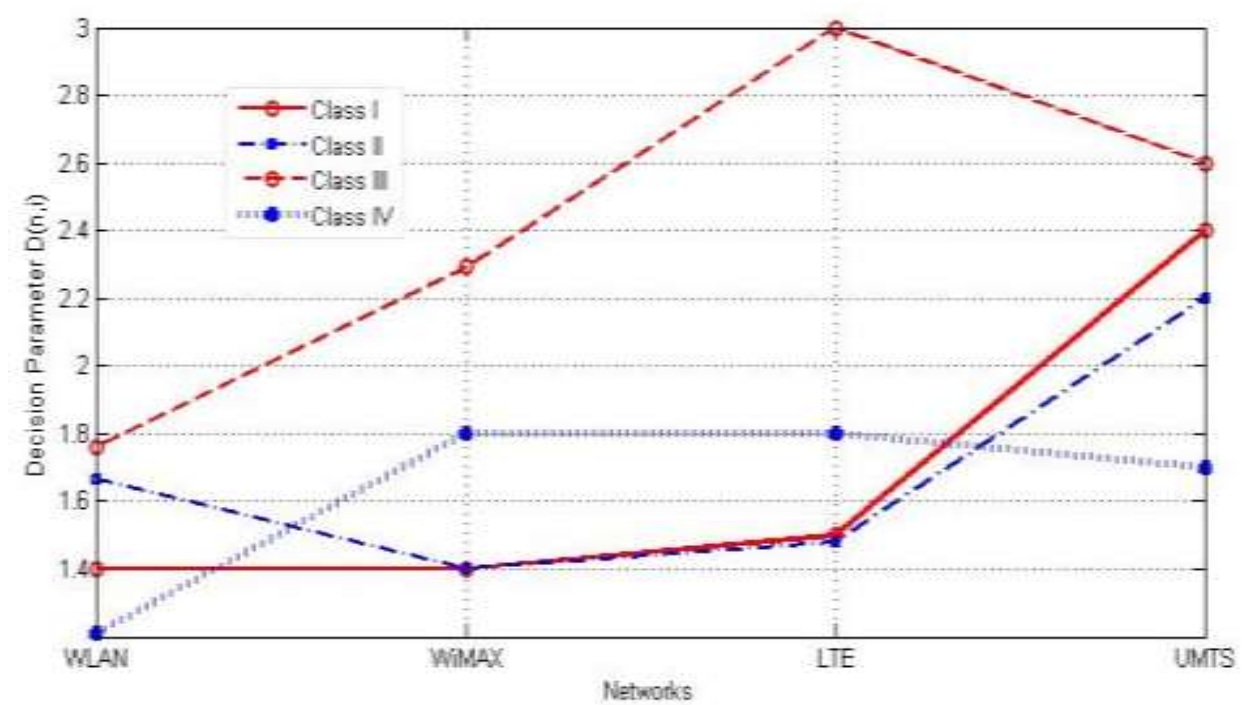

Figure 10. Decision Parameters for Service Class i

However, since sensory data is being sent over a long period of time, we have considered the issue of network cost as well. Here, to minimize our cost, we can also select an economically cheaper network such as WiFi or WLAN, if the decision parameters of these networks are above the threshold. We have left this choice upon the user. Such a freedom of choice using network cost, however, is only considered in non-emergency data. Similarly, it is assumed that the after a span of ten minutes the patient is required to have their ECG graph transmitted over to a doctor in another department of the hospital. Since now the class of data is changed, the algorithm selects UMTS to be the most suitable network for the current requirements. Hence, instead of depending on one network for all the requirements, we opted for choosing a network which fits our needs better.

\subsubsection{Throughput}

The proposed algorithm ensures high throughput as computed in Figure 11 for the networks in the simulation model. The throughput calculated here is done using the same scenarios as before. The throughput of the four networks are calculated when they were selected as serving network for general patient monitoring for the hundred patients. Here, Class IV data, i.e., sensory data such as blood pressure, heart rate etc., is being transmitted over to the nursing station. Since the number of handover in our algorithm is much less on a purely RSS based algorithm, the network is capable of staying connected for longer without disturbance, which contributes to higher throughput values in this system. 


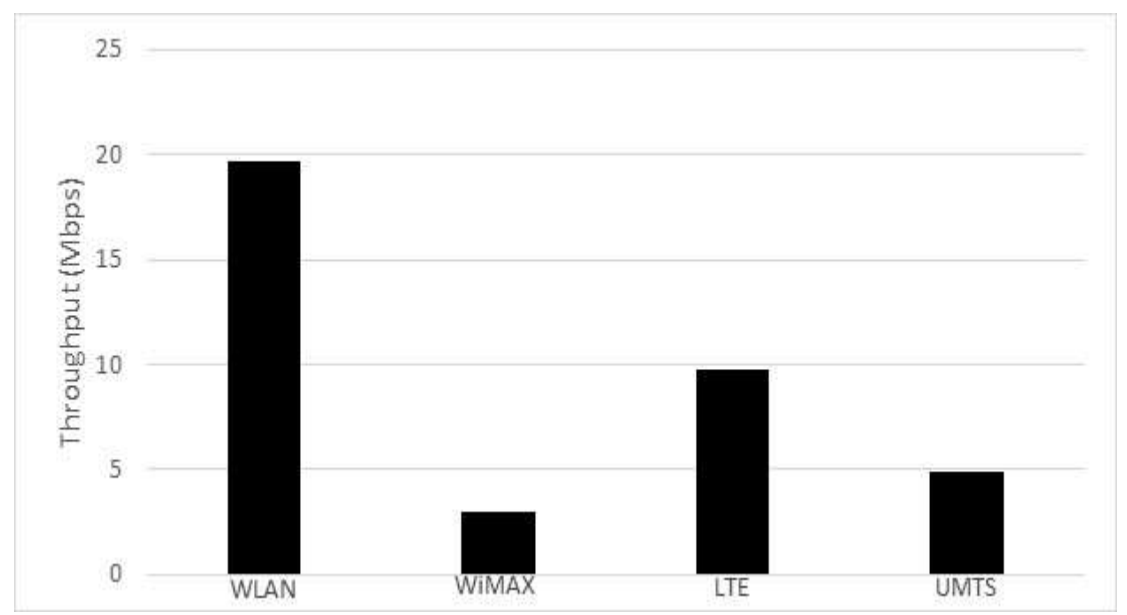

Figure 11. Throughput of the Available Networks

\subsubsection{Minimum Transfer Time}

Figure 12 compares the minimum transfer time (MTT) of the networks computed for all the services. For sensitive applications, such as patient data, a shorter transfer time translates into a better performance of the network. The less number of handovers executed by our algorithm, as observed in Figure 10 also contributes to less time devoted in performing handovers and thus less overall time is wasted in data transmission.

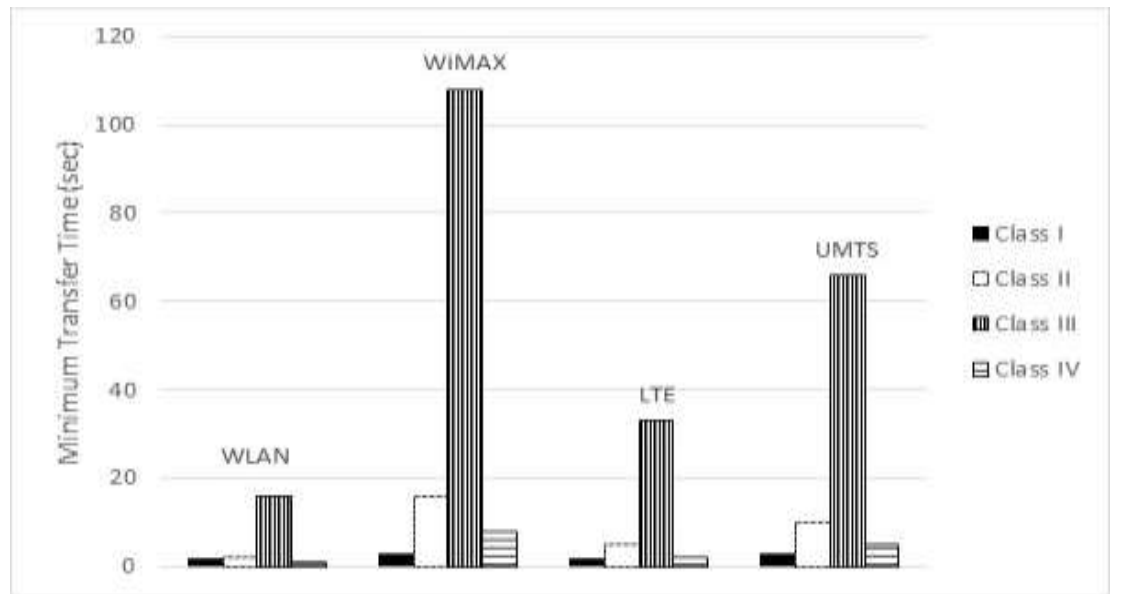

Figure 12. Minimum Transfer Time of the Networks for Different Class of Service Data

\section{Conclusion}

The paper proposes a novel Network selection based on the QoS needed as per the type of data to be sent from patient end to hospital/doctor. The simulations were carried out for widely used technologies such as WLAN, WiMAX, LTE and UMTS for healthcare services. Based on the simulation studies the most suitable network was selected to be accessed by the user for a particular type of data. The effectiveness of the proposed framework can be witnessed in minimising the number of handover through maximizing the user experience and maintaining the minimum QoS required for the transmission of data. It is important to note that through such framework one can provide a seamless connectivity which suffices the major requirement of such critical applications. 
Future will focus to have more categories of medical data so as to increase the accuracy of the proposed model and variability. The factor of patient mobility can be interesting aspect to be introduced further to shape our analysis to more real life situation.

\section{References}

[1] "Physicians per 1,000 people", Available at: https://data.worldbank.org/indicator/SH.MED.PHYS.ZS Data.worldbank.org. (2018).

[2] B. Potnuru, "Aggregate Availability of Doctors in India: 2014-2030", Indian Journal of public health, vol. 61, no. 3, (2017), pp. 182-187.

[3] T. Wu, F. Wu, J. M. Redoute and M. R. Yuce, "An Autonomous Wireless Body Area Network implementation towards IoT connected Healthcare applications,", IEEE Access J., vol. 5, (2017), pp. 11413-11422.

[4] M. Adnan, S. M. S. Hilles and W. M. S. Yafooz, "An Evolution to Next Generation Heterogeneous Cellular Networks", International Journal of Computer Science and Network Security, vol. 17, no. 3, (2017), pp. 251-255.

[5] P. Mather and B. Tso, "Classification methods for remotely sensed data", CRC press, Boca Raton, (2016).

[6] P. Keikhosrokiani, N. Zakaria, N. Mustaffa, T.C. Wan, M. I. Sarwar and K. Azimi, "Wireless networks in mobile healthcare", Edited S. Adibi, Springer International Publishing, Burwood, vol. 5, (2015), pp. 687-726.

[7] K. Cai and X. Liang, "Development of WI-FI Based Telecardiology Monitoring System”, Proceedings of the 2nd International Workshop on Intelligent Systems and Applications, Wuhan, China, (2010) May 22-23.

[8] P. Yadav, R. Agrawal and K. Kashish, "Performance Evaluation of ad hoc Wireless Local Area Network in Telemedicine Applications", Journal of Procedia Computer Science, vol. 125, (2018), pp. 267-274.

[9] D. Niyato, E. Hossain and J. Diamond, "IEEE 802.16/WiMAX-based broadband wireless access and its application for telemedicine/e-health services", IEEE Wireless Communications, vol. 14, no. 1, (2007), pp. 72-83.

[10] P. Goyal, D. Lobiyal and C. Katti, "Dynamic User Preference Based Network Selection for Vertical Handoff in Heterogeneous Wireless Networks", Wireless Personal Communications, vol. 98, no. 1, (2018), pp. 725-742.

[11] M. Salayma, A. Al-Dubai, I. Romdhani and Y. Nasser, "Wireless Body Area Network (WBAN): A Survey on Reliability, Fault Tolerance, and Technologies Coexistence", ACM Computing Surveys (CSUR), vol. 50, no. 1, (2017), pp. 1-38.

[12] K. Gündogdu and A. Çalhan, "An Implementation of Wireless Body Area Networks for Improving Priority Data Transmission Delay”, Journal of medical systems, vol. 40, no. 3, (2016), pp. 74-80.

[13] R. Punj and R. Kumar, "Technological aspects of WBANs for health monitoring: a comprehensive review", Wireless networks, vol. 24 no.152, (2018), pp. 1-33.

[14] M. Gondara1 and S. Kadam, "Requirements of vertical handoff mechanism in 4G wireless networks", International Journal of Wireless \& Mobile Networks, vol. 3, no. 2, (2011), pp. 18-27.

[15] X. Yan, Y. Sekercioglu and S. Narayanan, "A survey of vertical handover decision algorithms in Fourth Generation heterogeneous wireless networks", Computer networks., vol. 54, no. 11, (2010), pp. 18481863.

[16] A. M. Vegni, G. Tamea, T. Inzerilli and R. Cusani, "A combined vertical handover decision metric for QoS enhancement in next generation networks", Proceedings of 5th IEEE International Conference on Wireless and Mobile Computing, Networking and Communications, Marrakech, Morocco, (2009) October 12-14.

[17] N. Nasser, A. Hasswa and H. Hassanein, "Handoffs in fourth generation heterogeneous networks", IEEE Communications Magazine, vol. 44, no. 10, (2006), pp. 96-103.

[18] E. Navarro and V. Wong, "Comparison between vertical handoff decision algorithms for heterogeneous wireless networks", Proceedings of the 63rd IEEE Vehicular technology conference, Melbourne, Australia, (2006) May 7-10.

[19] H. Elhadj, J. Elias, L. Chaari and L. Kamoun, "Multi-Attribute Decision Making Handover Algorithm for Wireless Body Area Networks", Computer Comm. vol. 81, (2016), pp. 97-108.

[20] J. Zubairi, S. Misbahuddin and I. Tasadduq, "Emergency medical data transmission systems and techniques", Edited K. Khoumbati, IGI Global publisher, Hershey, PA, (2010), pp. 167-187

[21] IEEE 802.21 Working Group, "IEEE standard for local and metropolitan area networks-Part 21", Media independent handover, (2009).

[22] Y. Chen, "Equivalent Relation between Normalized Spatial Entropy and Fractal Dimension", arXiv preprint arXiv:1608.02054, (2016), pp. 1-28.

[23] M.C Chuah and Q. Zhang, "Design and Performance of 3G Wireless Networks and wireless LANS", Springer US Publisher, (2005). 
[24] N. Meghanathan, S. Boumerdassi, N. Chaki and D. Nagamalai, Editors, "Recent Trends in Networks and Communications", Verlag Berlin Heidelberg Publishers, Springer, (2010).

\section{Authors}

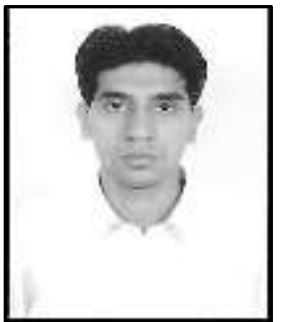

Piyush Yadav, received his graduation and post-graduation in Electronics and Communication Engineering from India. He is pursuing $\mathrm{Ph}$. D. in Electronics Engineering. $\mathrm{He}$ is working as Assistant Professor at GLBITM, Greater Noida, India. His major research areas include wireless communication and ICT in healthcare with specific interest in heterogeneous networks.

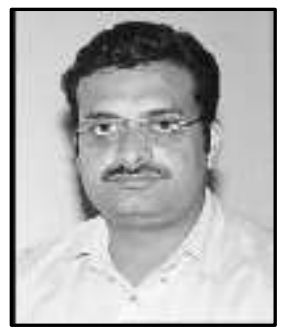

Dr. Rajeev Agrawal, received his graduation in Electronics Engineering and post-graduation in Systems Sciences from India. $\mathrm{He}$ has done Ph.D. in the area of Wireless communication from Jawaharlal Nehru Univ., New Delhi, India. He is working as Professor and Director at GLBITM, Greater Noida, India. His current areas of research are wireless networks and Ultrasound Medical Imaging.

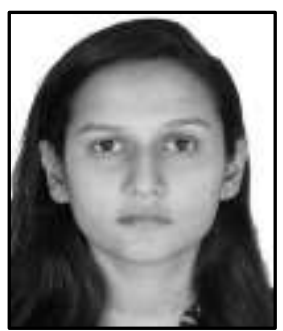

Komal Kashish, is pursuing her Master's degree in Communication Engineering at RWTH, Germany and working at Fraunhofer Institute for Communication, Information Processing and Ergonomics, Bonn, as a student worker. Her research interests include communications systems and network programming. 